\title{
Functional analysis of multiple nifB genes of Paenibacillus strains in synthesis of Mo-, Fe- and V-nitrogenases
}

\author{
Qin Li ${ }^{1,2}$, Haowei Zhang ${ }^{1}$, Liqun Zhang ${ }^{2}$ and Sanfeng Chen ${ }^{1 *}$ (1)
}

\begin{abstract}
Background: Biological nitrogen fixation is catalyzed by Mo-, V- and Fe-nitrogenases that are encoded by nif, vnf and anf genes, respectively. NifB is the key protein in synthesis of the cofactors of all nitrogenases. Most diazotrophic Paenibacillus strains have only one nifB gene located in a compact nif gene cluster (nifBHDKENX(orf1)hesAnifl). But some Paenibacillus strains have multiple nifB genes and their functions are not known.

Results: A total of 138 nifB genes are found in the 116 diazotrophic Paenibacillus strains. Phylogeny analysis shows that these nifB genes fall into 4 classes: nifBI class including the genes (named as nifB1 genes) that are the first gene within the compact nif gene cluster, nifBll class including the genes (named as nifB2 genes) that are adjacent to anf or vnf genes, nifBlll class whose members are designated as nifB3 genes and nifBIV class whose members are named as nifB4 genes are scattered on genomes. Functional analysis by complementation of the $\triangle$ nifB mutant of $P$. polymyxa which has only one nifB gene has shown that both nifB1 and nifB2 are active in synthesis of Mo-nitrogenase, while nifB3 and nifB4 genes are not. Deletion analysis also has revealed that nifB1 of Paenibacillus sabinae T27 is involved in synthesis of Mo-nitrogenase, while nifB3 and nifB4 genes are not. Complementation of the P. polymyxa $\triangle$ nifBHDK mutant with the four reconstituted operons: nifB 1anfHDGK, nifB2anfHDGK, nifB1VnfHDGK and nifB2VnfHDGK, has shown both that nifB1 and nifB2 were able to support synthesis of Fe- or V-nitrogenases. Transcriptional results obtained in the original Paenibacillus strains are consistent with the complementation results.

Conclusions: The multiple nifB genes of the diazotrophic Paenibacillus strains are divided into 4 classes. The nifB 1 located in a compact nif gene cluster (nifBHDKENX(orf1)hesAnifl) and the nifB2 genes being adjacent to nif or anf or vnf genes are active in synthesis of Mo-, Fe and V-nitrogenases, but nifB3 and nifB4 are not. The reconstituted anf system comprising 8 genes (nifBanfHDGK and nifXhesAnifl) and vnf system comprising 10 genes (nifBVnfHDGKEN and nifXhesAnifV) support synthesis of Fe-nitrogenase and V-nitrogenase in Paenibacillus background, respectively.
\end{abstract}

Keywords: Paenibacillus, nifB gene, Mo-nitrogenase, Alternative nitrogenases

\footnotetext{
*Correspondence: chensf@cau.edu.cn

${ }^{1}$ State Key Laboratory for Agrobiotechnology, College of Biological Sciences, China Agricultural University, Beijing 100193, People's Republic of China

Full list of author information is available at the end of the article
}

\section{Background}

Biological nitrogen fixation, a process unique to some bacteria and archaea (called diazotrophs), is catalyzed by nitrogenase and plays an important role in world agriculture [1]. There are three known nitrogenase designated as the Mo-nitrogenase, V-nitrogenase and Fe-nitrogenase that are encoded by nif, vnf, and anf, respectively [2]. Nitrogen fixation is mainly catalyzed by Mo-nitrogenase, which is found in all diazotrophs. In addition to

(C) The Author(s) 2021. Open Access This article is licensed under a Creative Commons Attribution 4.0 International License, which permits use, sharing, adaptation, distribution and reproduction in any medium or format, as long as you give appropriate credit to the original author(s) and the source, provide a link to the Creative Commons licence, and indicate if changes were made. The images or other third party material in this article are included in the article's Creative Commons licence, unless indicated otherwise in a credit line to the material. If material is not included in the article's Creative Commons licence and your intended use is not permitted by statutory regulation or exceeds the permitted use, you will need to obtain permission directly from the copyright holder. To view a copy of this licence, visit http://creativecommons.org/licenses/by/4.0/. The Creative Commons Public Domain Dedication waiver (http://creativecommons.org/ publicdomain/zero/1.0/) applies to the data made available in this article, unless otherwise stated in a credit line to the data. 
Mo-nitrogenase, some possess either of alternative Fenitrogenase and V-nitrogenase, or both. Each nitrogenase contains two components, a catalytic protein and a reductase [3-5]. For Mo-nitrogenase, MoFe protein is the catalytic protein and Fe protein is the reductase. The MoFe protein is an $\alpha_{2} \beta_{2}$ heterotetramer (encoded by nifD and nifK) that contains two metal clusters: FeMo-co, a [Mo-7Fe-9S-C-homocitrate] cluster which serves as the active site of $\mathrm{N}_{2}$ binding and reduction and the P-cluster, a [8Fe-7S] cluster which shuttles electrons to FeMoco. The Fe protein (encoded by nifH) is a homodimer bridged by an intersubunit [4Fe-4S] cluster that serves as the obligate electron donor to the MoFe protein [6-8]. Like Mo-nitrogenase, alternative nitrogenases comprise an electron-delivery Fe protein (encoded by anfH in $\mathrm{Fe}-$ nitrogenase and encoded by $v n f H$ in $\mathrm{V}$-nitrogenase). The FeFe protein of Fe-nitrogenase encoded by anfDK and the VFe protein of V-nitrogenase encoded by $v n f D K$ are homologous to the MoFe protein of Mo-nitrogenase. The alternative nitrogenases have either FeFe-co or FeV-co at the active site and also include an additional subunit (AnfG or VnfG) encoded by anfG or $v n f G$ [9]. The FeFeco is analogous to FeMo-co except for containing Fe in place of Mo [10], but FeV-co is a [V-7Fe-8S-C-homocitrate] cluster which replaces Mo with $\mathrm{V}$ and lacks one $\mathrm{S}$ compared to FeMo-co [11].

NifB has been demonstrated to be essential for the synthesis of all nitrogenases. NifB is a radical S-adenosyl methionine (SAM) enzyme that catalyzes the formation of NifB-co, a [8Fe-9S-C] cluster which is a common precursor for the syntheses of FeMo-co of Mo-nitrogenase, $\mathrm{FeV}$-co of V-nitrogenase and FeFe-co of Fe-nitrogenase [12-14]. NifB-co is subsequently transferred to the scaffold protein NifEN, upon which mature cofactor is synthesized. The NifX protein is known to bind NifB-co and involved in NifB-co transfer [15].

The number, structure and properties of nifB genes show some variation among different diazotrophs. Azotobacter vinelandii and Rhodopseudomonas palustris possess only one nifB gene that is responsible for three types of nitrogenases and mutation of nifB gene led to loss of all nitrogenases activities [16, 17]. Rhodobacter capsulatus with Mo-nitrogenase and Fe-nitrogenase carries two nifB genes that are located in two nif gene clusters [18] and either one of the two nifB genes was sufficient for nitrogen fixation via the Mo-dependent or Fe-dependent nitrogenase [19]. The cyanobacterium Anabaena variabilis ATCC 29,413 has two nifB genes for synthesis of two Mo-nitrogenases, but nifB1 is specifically expressed in heterocysts and nifB2 is specifically expressed in vegetative cells [20]. On the basis of $\mathrm{NifB}$ domain architecture, the NifB proteins are divided into three subfamilies [21, 22]. The first NifB subfamily has an $\mathrm{N}$-terminal SAM-radical domain linked to a C-terminal NifX-like domain. A major of NifB proteins from Bacteria domain (e.g. A. vinelandii and Klebsiella oxytoca) belong to the first NifB subfamily. The second NifB subfamily contains a stand-alone SAM-radical domain and is found in Bacteria and Archaea domains. The third NifB subfamily has three domains including a NifN-like domain, a SAM-radical domain and a C-terminal NifX-like domain and is found in Clostridium species.

The Paenibacillus genus of the Firmicutes phylum is a large one that currently comprises 254 validly named species (https://www.bacterio.net/paenibacillus.html), more than 20 of which have the nitrogen fixation ability [23]. Comparative genome sequence analysis of 15 diazotrophic Paenibacullus strains have revealed that a compact nif gene cluster comprising 9-10 genes (nifB nifH nifD nifK nifE nif $N$ nif $X$ (orf1) hesA nifV) encoding Mo-nitrogenase is conserved in the $\mathrm{N}_{2}$-fixing Paenibacillus genus [24]. The 9 genes (nifBHDKENXhesAnifV) in Paenibacillus polymyxa WLY78 are organized as an operon under control of a $\sigma^{70}$ dependent promoter located in front of nifB gene [25]. In addition to the nif gene cluster, additional nif genes or anf or $v n f$ genes are found in some diazotrophic Paenibacillus spp. For examples, Paenibacillus sabinae T27 has multiple nifB, nifH, nifE and nifN genes [26]. Paenibacillus forsythia T98 and Paenibacillus sophorae S27 have additional nif and anfDHGK genes, Paenibacillus zanthoxyli JH29 and Paenibacillus durus (previously called as Paenibacillus azotofixans) ATCC 35681 contain additional nif and $v n f D H G K E N$ genes [24]. Notably, in addition to the nifB gene in the compact nif gene cluster comprising 9-10 genes (nifB nifH nifD nifK nifE nifN nifX (orf1) hesA nifV) encoding Mo-nitrogenase, multiple nifB genes are found in some Paenibacillus species that carry additional nif genes or anf genes or $v n f$ genes [24, 26]. However, functions of the multiple nifB genes are not known. In this study, we analyzed the distribution and phylogeny of the 138 putative NifB proteins from 116 diazotrophic Paenibacillus strains. All nifB genes in Paenibacillus fall into 4 classes: nifBI, nifBII, nifBIII and nifBIV. We demonstrate that only nifBI and nifBII are functional in synthesis of Mo-, Fe- and V-nitrogenase. The nifBIII and nifBIV may be not involved in nitrogen fixation. In addition, the reconstituted anf system comprising 8 genes (nifBanfHDGK and nifXhesAnifV) and $v n f$ system comprising 10 genes (nifBvnfHDGKEN and nifXhesAnifV) supported synthesis of Fe-nitrogenase and V-nitrogenase in Paenibacillus background, respectively. Our study will provide guidance for engineering nitrogenase into heterologous hosts. 


\section{Results}

\section{Classification of nifB genes of Paenibacillus genus}

Here, the nitrogen fixation genes in the genomes of the 116 diazotrophic Paenibacillus strains taken from the RefSeq database are comparatively analyzed (Additional file 1: Table S1). A compact nif gene cluster composed of 9-10 genes (nifBHDKENX(orf1)hesAnifV) is conserved in all of the diazotrophic strains, in agreement with the previous studies [24]. In addition to the compact nif gene cluster encoding Mo-nitrogenase, 9 strains have additional anfHDGK encoding Fe-nitrogenase and 3 strains have additional $\nu n f H D G K E N$ encoding V-nitrogenase.

A total of $138 \mathrm{NifB}$ putative sequences are found in the 116 diazotrophic Paenibacillus strains. According to the nifB sequence similarity, the nifB genes were divided into 4 classes. The nifBI class includes the nif $B$ genes (named as nifB1 genes) that are the first gene in the compact nif gene cluster comprising 9-10 genes (nifB nifH nifD nifK nifE nifN nifX (orf1) hesA nifV) and the genes linked to another nifH. The nifBII class includes these genes (named as nifB2 genes) that are linked to additional copies of nifENXorf(fer) genes preceding anfHDGK or additional copies of nifENXorforf genes preceding vnfHDGKEN or orforf preceding $v n f H D G K E N$. The genes (named as nifB3) of nifBIII class and the genes (named as nifB4) of nifBIV are scattered at different locations of genomes.

Of the 116 diazotrophic Paenibacillus strains, 105 strains have only one nifB and 11 strains have 2-4 nifB genes. Paenibacillus polymyxa WLY78 is a representative that has only a nifB1 located in the compact nif gene cluster consisting of 9 genes (nifBHDKENXhesAnifV) encoding Mo-nitrogenase (Fig. 1 and Additional file 1: Table S1). Paenibacillus sabinae T27 is a representative strain with three nifB genes (nifB1, nifB3 and nifB4), but contained only Mo-nitrogenase. For the strains with both Mo- and V-nitrogenases, Paenibacillus zanthoxyli JH29 has nifB1, nifB2 and nifB3, but Paenibacillus durus DSM 1735 has nifB2, nifB3 and 2 copies of nifB1: one being located in the compact nif cluster and the other being linked to another nifH. For the strains with both Mo- and Fe-nitrogenases, Paenibacillus forsythiae T98 has three nifB genes (nifB1, nifB2 and nifB3), whereas Paenibacillus sophorae S27 has four nifB genes (nifB2, nifB3, and 2 copies of nifB1). The other 4 strains (Paenibacillus borealis FSL H70744, Paenibacillus sp. FSL H7-0357,
Paenibacillus sp. HW567 and Paenibacillus camerounensis G4) with both Mo- and Fe-nitrogenases possess only one nifB gene. Organization of the nifB genes and other nitrogen fixation genes from 17 representatives of Paenibacillus strains is shown in Fig. 1.

\section{Phylogeny and structure of Paenibacillus NifB proteins}

Here, 138 putative NifB sequences from 116 diazotrophic Paenibacillus strains are used to construct a phylogenetic tree, with 11 NifB sequences from 10 diazotrophs (A. vinelandii, K. oxytoca, Bradyrhizobium japonicum, Clostridium kluyveri, Dehalobacter sp., Kyrpidia spormannii, Methanosarcina acetivorans, Methanococcus maripaludis, Frankia sp. EAN1pec, Nostoc sp. PCC 7120) as control (Fig. 2 and Additional file 1: Table S1). The phylogenetic tree has shown that all Paenibacillus putative NifB proteins form a large class which is separated from the NifB proteins from other diazotrophs. The data suggest that all Paenibacillus putative nifB genes have a common ancestor. The Paenibacillus putative NifB proteins are divided into 4 classes: NifBI, NifBII, NifBIII and NifBIV, which corresponded to the 4 nifB classes that are classified on basis of nifB sequence similarities. The NifB1, NifB2, NifB3 and NifB4 proteins corresponded to NifBI, NifBII, NifBIII and NifBIV classes, respectively. Phylogeny analyses have shown that the NifB1 proteins are emerged firstly in the diazotrophic Paenibacillus species, and NifB2, NifB3 and NifB4 may result from gene duplication.

Protein structure analysis showed that Paenibacillus NifB1, NifB2 and NifB4 have the same structure composed of an $\mathrm{N}$-terminal SAM-radical domain and a C-terminal NifX-like domain. Most NifBIII members possesses the two domains, but the NifB3 proteins from the 2 strains (P. zanthoxyli $\mathrm{JH} 29$ and $P$. durus DSM 1735) have only a SAM-radical domain. The Paenibacillus NifB1, NifB2, NifB3 and NifB4 proteins that possess both domains are composed of 427-505 amino acids (Additional file 1: Table S1) and have similarity ( $>57 \%$ ) at amino acid levels. These proteins have a number of conserved motifs in the SAM-radical domain, including HPC motif, $\mathrm{Cx}_{3} \mathrm{Cx}_{2} \mathrm{C}$ motif, ExRP motif, AGPG motif, TxTxN motif and $\mathrm{Cx}_{2} \mathrm{CRxDAxG}$ (Fig. 2). However, the NifB3 proteins of $P$. zanthoxyli JH29 and P. durus DSM 1735 have only a SAM-radical domain that lacks the Cx2CRxDAxG motif. Sequence alignment of 13 NifB

(See figure on next page.)

Fig. 1 Genetic organization of the nifB loci and other nif, anf, vnf genes in $\mathrm{N}_{2}$-fixing Paenibacillus strains. The compact nif gene cluster comprising contiguous 9-10 genes nifBHDKENX(orf1)hesAnifV. The anf genes are marked with yellow color and the vnf genes are marked with apricot yellow. The nifB genes are shown in magenta. The nifX-like genes whose predicted products show high sequence similarity with the C-terminal domain of NifB are shown in pink 
sis

$\Rightarrow \Rightarrow \Rightarrow \Rightarrow$

sis

$\rightarrow \Rightarrow \Rightarrow$

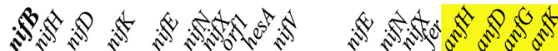

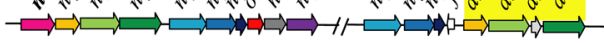

sis

$\Rightarrow$

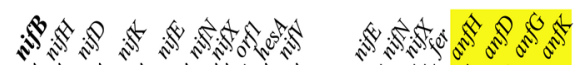

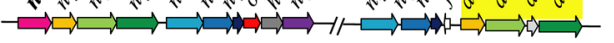

s.s.

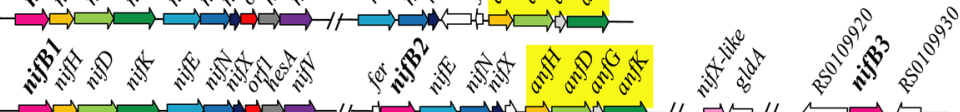

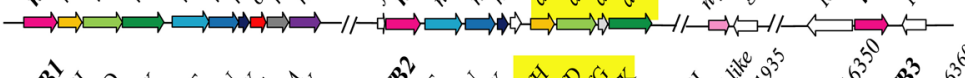

然

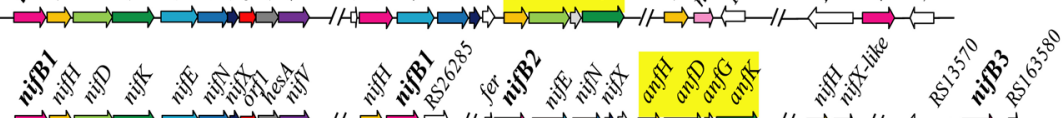

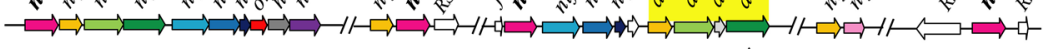

sis

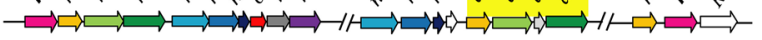

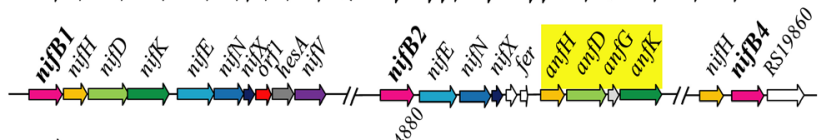

$\Rightarrow \rightarrow \Rightarrow$

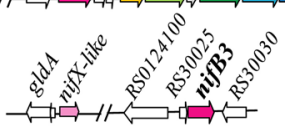

s

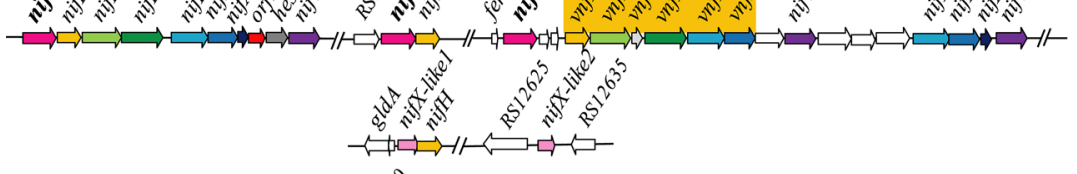

s.

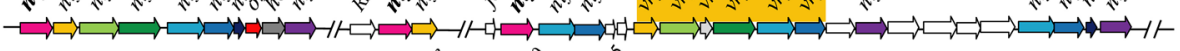

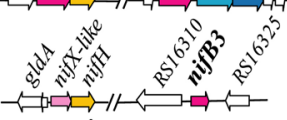

(n)

s.s.

sis

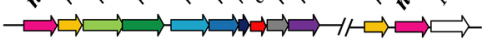

Fig. 1 (See legend on previous page.)

\section{P. polymyxa WLY78}

P. macerans 8244

P. borealis FSL H7-0744

Paenibacillus sp. FSL H7-0357

P. camerounensis G4

Paenibacillus sp. HW567

P. forsythia $\mathrm{T} 98$

P. stellifer DSM 14472

P. sophorae $\mathbf{S 2 7}$

P. riograndensis SBR5

P. borealis DSM 13188

P. zantofixans JH29

P. durus ATCC 35681

P. durus DSM 1735

P. sabinae T27

P. rhizophilus 7197

Paenibacillus sp. IHB B 3415 


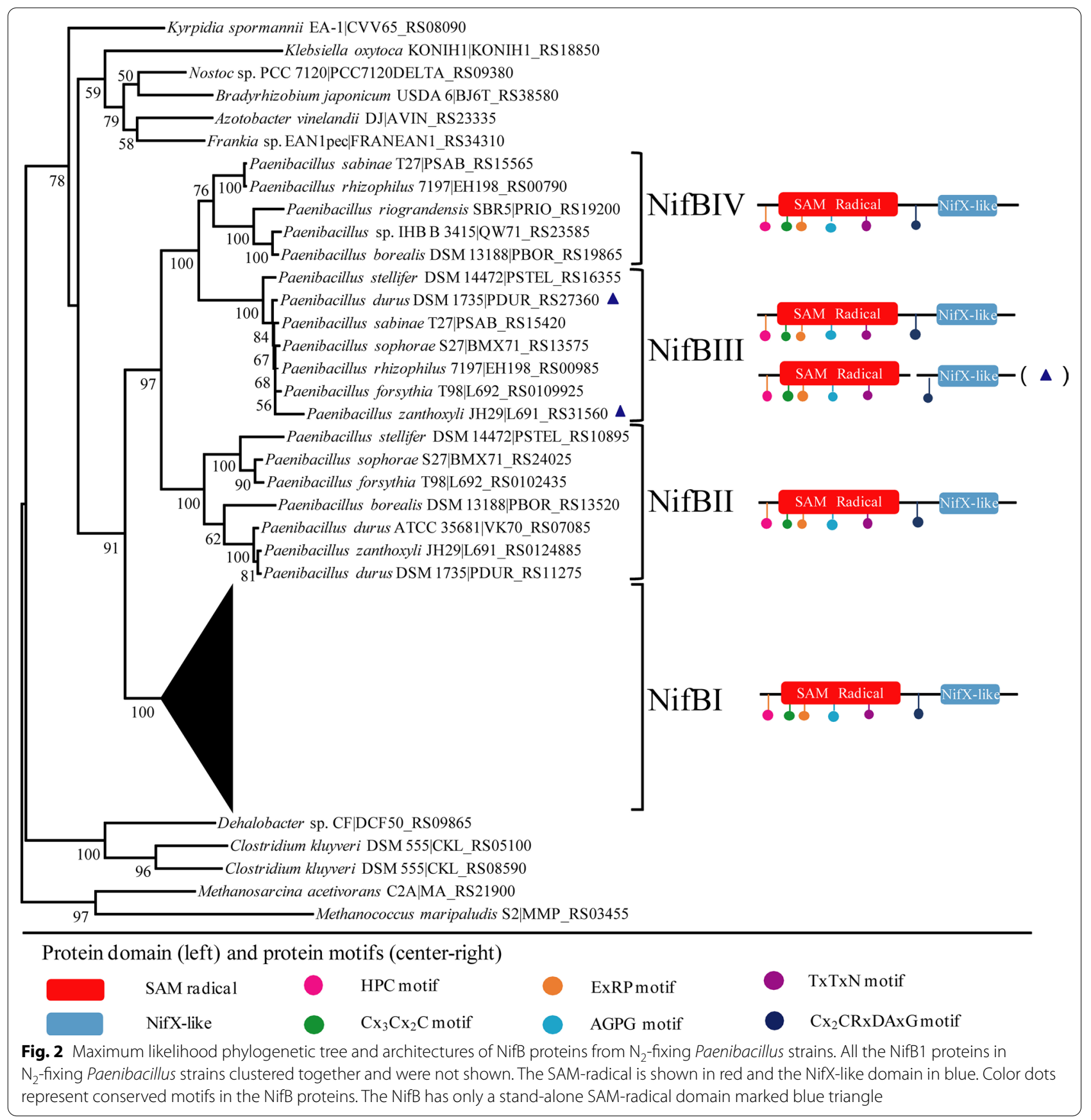

proteins including NifB1, NifB2, NifB3 and NifB4 from 4 representatives of Paenibacillus strains (P. polymyxa WLY78, P. sabinae T27, P. forsythia T98 and $P$. zanthoxyli $\mathrm{JH} 29$ ) is shown in Additional file 1: Figure S1.

\section{Transcription analysis of multiple nifB genes in medium containing only Mo or Fe or $\mathbf{V}$}

As described above, $P$. sabinae T27 with only Mo-nitrogenase has NifB1, NifB3 and NifB4, P. zanthoxyli JH29 with both Mo- and V-nitrogenases has NifB1, NifB2 and NifB3 and P. forsythiae T98 with both Mo- and Fenitrogenases possesses NifB1, NifB2 and NifB3. The three species $P$. sabinae T27, $P$. forsythia T98 and $P$. zanthoxyli $\mathrm{JH} 29$ were used to investigate the transcriptions of the multiple nifB genes under different conditions by RT-qPCR. Paenibacilllus sabinae T27 was cultivated in Mo-dependent $\mathrm{N}_{2}$-fixing condition, while P. forsythia $\mathrm{T} 98$ and P. zanthoxyli $\mathrm{JH} 29$ were cultivated 

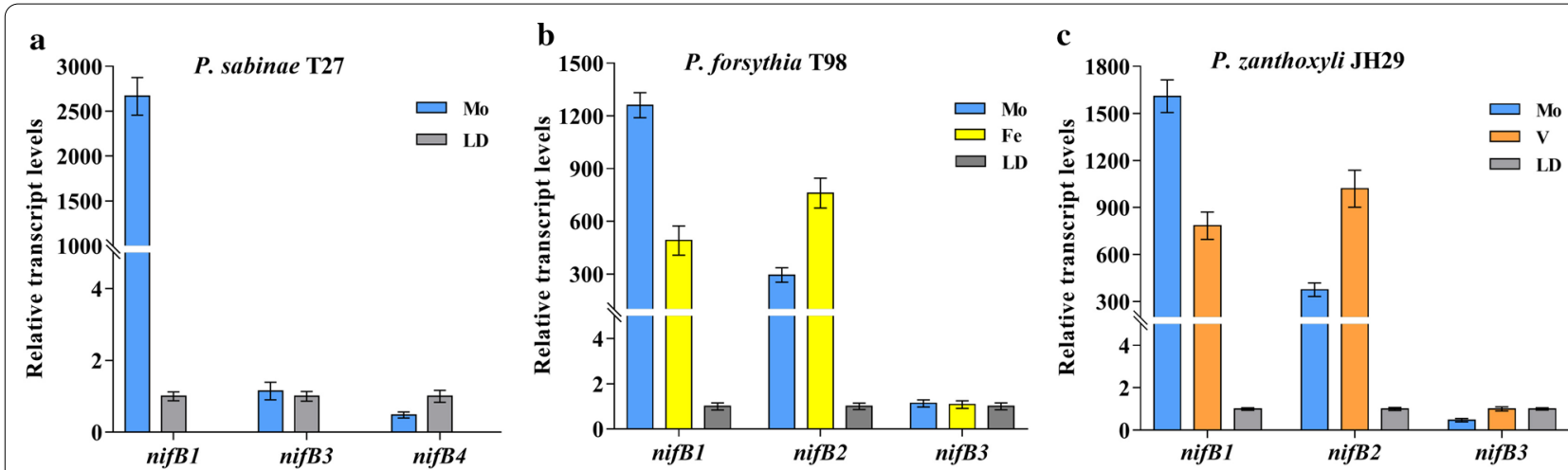

Fig. 3 Transcription profile of the multiple nifB genes from P. sabinae T27(a), P. forsythia T98(b) and P. zanthoxyli JH29(c). RT-qPCR analysis of the relative transcript levels of the nifB genes in these Paenibacillus species grown in Mo-dependent, Fe-dependent and V-dependent nitrogen fixation conditions, with non-nitrogen fixing conditions of N-rich (LD medium) cultures as negative controls. The data are the mean of three biological replicates

in Mo-dependent and Fe-dependent or V-dependent $\mathrm{N}_{2}$-fixing condition, respectively, with non- $\mathrm{N}_{2}$-fixing condition of N-rich (LD medium) cultures as negative controls (Fig. 3). For P. sabinae T27, the transcription level of nifB1 exhibited more than 2000-fold of increase under Mo-dependent $\mathrm{N}_{2}$-fixing condition compared to under non- $\mathrm{N}_{2}$-fixing condition, but the transcripts from nifB3 and nifB4 showed no differences under both conditions (Fig. 3a). For $P$. forsythia T98 grown under both Mo-dependent and Fe-dependent condition, both nifB1 and nifB2 genes were highly transcribed, but nifB3 was not induced by $\mathrm{N}_{2}$-fixing condition. The transcript level of nifB1 was much higher in Mo-dependent condition than in Fe-dependent condition, while the transcript level of nifB2 was higher in Fe-dependent condition than in Mo-dependent condition (Fig. 3b). For P. zanthoxyli $\mathrm{JH} 29$ grown under both Mo-dependent and V-dependent conditions, the transcription of both nifB1 and nifB2 genes were activated, but nifB3 showed no differences in its expression under test conditions. The transcript level of nifB1 was higher in Mo-dependent condition than in V-dependent condition, while the transcript level of nifB2 was higher in V-dependent condition than in Modependent condition (Fig. 3c). These results indicate that the nifB1 and nifB2 may be selectively expressed according to metal availability.

\section{Functional analysis of multiple nifB genes in synthesis of Mo-nitrogenase}

The nifB deletion mutant $(\Delta$ nifB) of $P$. polymyxa WLY78 was here constructed by using recombination method as described in materials and methods. The $P$. polymyxa $\triangle$ nifB mutant completely lost its nitrogenase activity and complementation by its nifB gene carried in a plasmid restored the nitrogenase activity (Fig. 4a).
Thus, P. polymyxa $\Delta$ nifB mutant was used as a host for complementation to investigate the functionality of the multiple nifB genes. Each nifB gene from $P$. sabinae T27, P. forsythia T98 and P. zanthoxyli JH29 was cloned into a low-copy plasmid pRN5101[27, 28], in which the expression of these nifB genes were driven under the control of the nifB promoter of $P$. polymyxa (details are provided in materials and methods). Among the 3 nifB genes of $P$. sabinae T27, only the nifB1 can effectively restore the nitrogenase activity of the $P$. polymyxa $\triangle$ nifB mutant, showing that the nifB1 gene was transcribed under nitrogen fixation condition and the translated NifB1 was functional. Both nifB1 and nifB2 from $P$. forsythia T98 or P. zanthoxyli JH29 can effectively restore nitrogenase activity of the $P$. polymyxa $\triangle$ nifB mutant, but the nifB3 from $P$. forsythia T98 or $P$. zanthoxyli $\mathrm{JH} 29$ can not restore activity. The result suggests that both nifB1 and nifB2 are functional in synthesis of Mo-nitrogenase, but nifB3 product was not active.

To further examine the role of the multiple nifB genes, attempts to inactivate the nifB genes were made. Three single deletion mutants $\triangle$ nifB $1, \Delta$ nifB3 and $\triangle n i f B 4$ of $P$. sabinae T27 were successfully constructed. Deletion of nifB1 resulted to complete loss of nitrogenase activity. Whereas, the nitrogenase activities of $\triangle n i f B 3$ or $\triangle$ nifB4 mutants were similar as that in wildtype P. sabinae T27 (Fig. 4b). The data are consistent with the above described qRT-PCR and heterologous complementation results, confirming that both nifB3 and nifB4 are not involved in nitrogen fixation. However, attempts to inactivate the nifB genes of $P$. forsythia T98 and P. zanthoxyli JH29 were not successful, due to hardness of genetic transformation in these strains. 

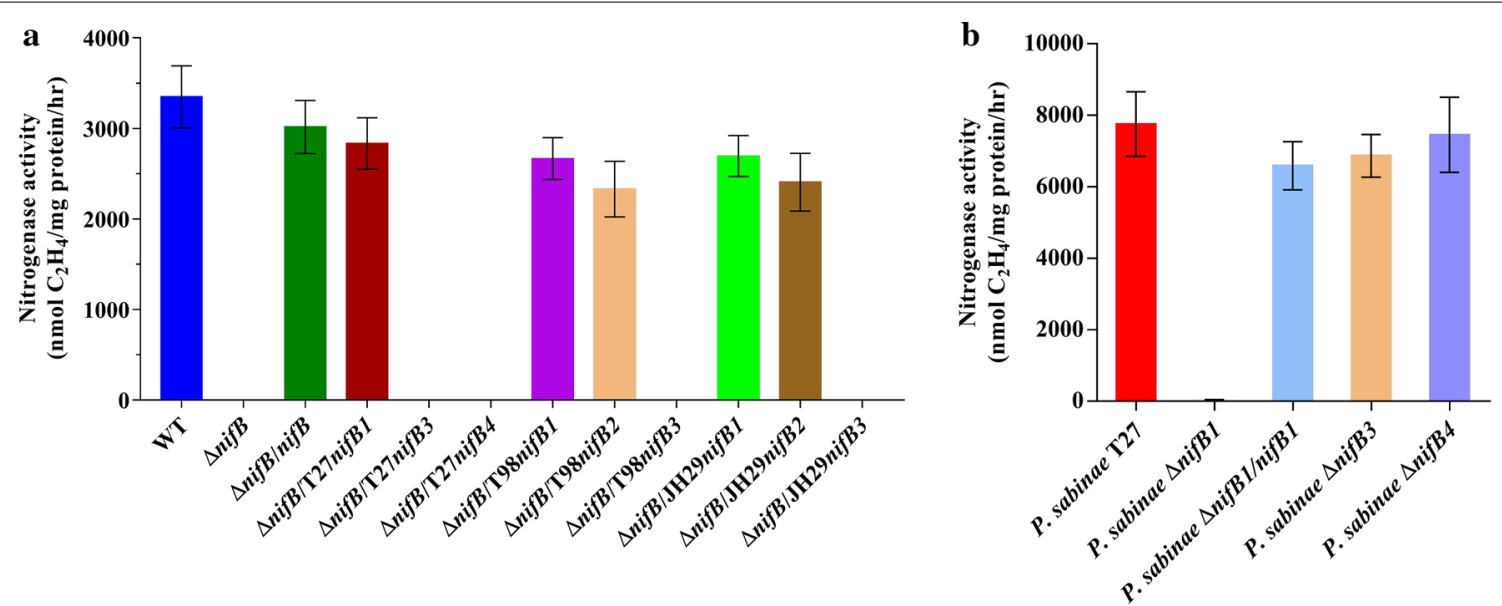

Fig. 4 The nitrogenase activities of the nifB deletion mutants and their complementary strains in Mo-dependent nitrogen fixation conditions. a Nitrogenase activities of the P. polymyxa $\triangle$ nifB mutant and its complementary strains. b Nitrogenase activities of WT (P. sabinaeT27), deletion mutants $\triangle$ nifB $1, \triangle$ nifB3, $\triangle$ nifB4 and complementary strain $\triangle$ nifB $1 / n i f B 1$. The nitrogenase activity was measured by acetylene reduction assay when bacterial cells were grown anaerobically in nitrogen limited medium containing Mo. Error bars indicate the SD observed from at least three independent experiments

\section{Functional analysis of nifB1 and nifB2 genes in synthesis of Fe- and V-nitrogenases}

In order to investigate whether the nifB1 and nifB2 from $P$. forsythia T98 and P. zanthoxyli JH29 were active in synthesis of Fe-nitrogenase and V-nitrogenases, the $\triangle n i f B H D K$ and $\triangle$ nifBHDKEN mutants of $P$. polymyxa WLY78 which lost the ability to synthesize Mo-nitrogenase were constructed. As shown in Fig. 5, the nifB$H D K$ and nifBHDKEN of $P$. polymyxa WLY78 carried in plasmid could restore the nitrogenase activity to $90 \%$ wild-type level in the complementary strain ( $\triangle$ nifBHDK/ nifBHDK) and ( $\triangle$ nifBHDKEN/nifBHDKEN), suggesting that the mutants can be used as a host for complementation study of alternative nitrogenases.

Two new operons nifB1anfHDGK and nifB2anfHDGK of $P$. forsythia T98 under the control of the $P$. polymyxa WLY78 nifB promoter were constructed (Fig. 5). Each of the reconstituted nifB1anfHDGK and nifB2anfH$D G K$ operons of $P$. forsythia T98 carried in the recombinant plasmids can enable $P$. polymyxa $\triangle$ nifBHDK mutant to have nitrogenase activity in medium containing Fe and lacking Mo. The data suggest that either
nifB1 or nifB2 together with anfHDGK of $P$. forsythia can support synthesis of Fe-nitrogenase in the heterologous host $P$. polymyxa which originally has only Mo-nitrogenase system. Furthermore, in order to investigate whether nifE and nif $N$ genes (designed nifE2 and nifN2 genes) preceding anfHDGK of P. forsythia T98 were functional, another new operon nifB2E2N2anfH$D G K$ of $P$. forsythia T98 was constructed (Fig. 5). Then, nifB2E2N2anfHDGK and nifB2anfHDGK carried in the recombinant plasmids are individually used to complement $\triangle$ nifBHDKEN mutant of P. polymyxa WLY78. As shown in Fig. 5, either nifB2E2N2anfHDGK or nifB2anfHDGK can support $\triangle$ nifBHDKEN mutant of $P$. polymyxa WLY78 to have nitrogenase activity in medium containing Fe and lacking Mo. Like the P. forsythia T98 that was capable of diazotrophic growth, the reconstituted nifB/anf-complemented strains can grow in liquid media with dinitrogen as the sole nitrogen source (Fig. $\mathrm{S} 2$ ). The results indicated that that nifEN is not necessary for the biosynthesis and the reconstituted anf system composed of 8 genes (nifBanfHDGK of $P$. forsythia

\section{(See figure on next page.)}

Fig. 5 Schematic map and nitrogenase activity of the $\triangle$ nifBHDK and $\triangle$ nifBHDKEN mutants of P. polymyxa and the complementary strains carrying nifB1anfHDGK, nifB2anfBHDGK, nifB2E2N2anfBHDGK of P. forsythia T98, respectively and the complementary strains carrying nifB 1vnfHDGK, nifB2VnfHDGK, nifB2VnfHDGKEN of P. zanthoxyli JH29, respectively. a Schematic map of the P. polymyxa $\triangle$ nifBHDK and P. polymyxa $\triangle$ nifBHDKEN mutants and the complementary strains. $\mathbf{b}$ The nitrogenase activity of the P. polymyxa $\triangle$ nifBHDK and P. polymyxa $\triangle$ nifBHDKEN mutants and the complementary strains. Activity was measured by acetylene reduction assay. The complementary strains carrying nifB1anfHDGK, nifB2anfBHDGK and nifB2E2N2anfBHDGK were cultivated in Fe-dependent conditions. The complementary strains carrying nifB $1 v n f H D G K$, nifB2VnfHDGK and nifB2VnfHDGKEN were cultivated in V-dependent conditions. Error bars indicate the SD observed from at least three independent experiments 


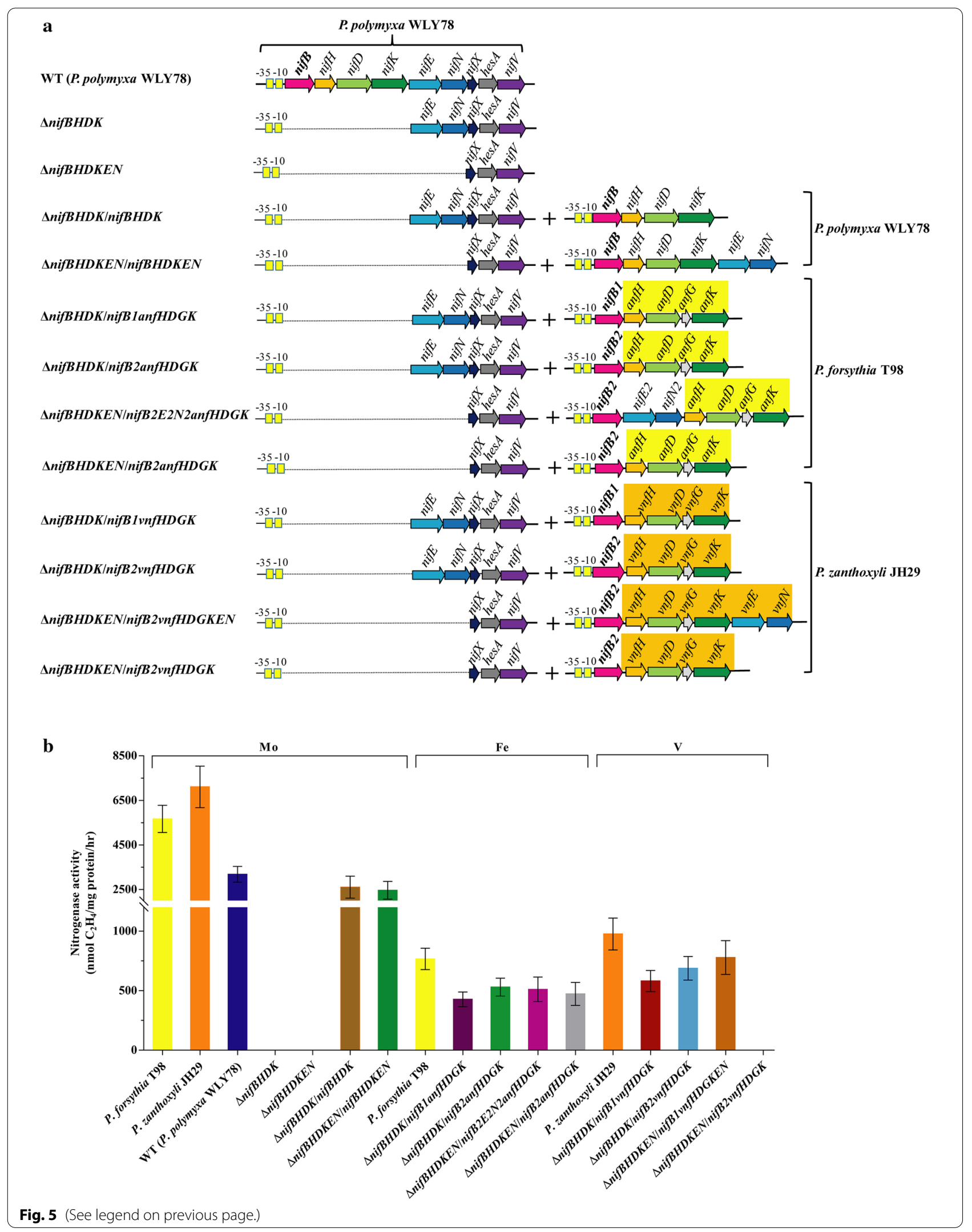


T98 and nifXhesAnifV of P. polymyxa WLY78) can support synthesis of Fe-nitrogenase to fix nitrogen.

Similarly, two new operons nifB1vnfHDGK and nifB$2 v n f H D G K$ of $P$. zanthoxyli $\mathrm{JH} 29$ under the control of the nifB promoter of $P$. polymyxa WLY78 were constructed (Fig. 5a). Each of the nifB1vnfHDGK and nifB2vnfHDGK operons of $P$. zanthoxyli JH29 carried in the recombinant plasmids can enable $P$. polymyxa $\triangle$ nifBHDK mutant to have nitrogenase activity in medium containing $\mathrm{V}$ and lacking Mo (Fig. 5b). The data suggest that either of nifB1 or nifB2 together with vnfHDGK of P. zanthoxyli $\mathrm{JH} 29$ can support synthesis of V-nitrogenase. Furthermore, a new operon comprising nifB2 and $v n f H D G K E N$ under the control of the nifB promoter of $P$. polymyxa WLY78 was constructed. The reconstituted operons nifB2vnfHDGKEN and nifB2vnfHDGK of P. zanthoxyli JH29 are individually used to complement $\triangle$ nifBHDKEN mutant of $P$. polymyxa WLY78. The operon nifB2vnfHDGKEN can effectively enable $\triangle$ nifBHDKEN mutant of $P$. polymyxa WLY78 to synthesize V-nitrogenase (Fig. 5). Our data demonstrate that the reconstituted $v n f$ system with vnfEN exhibited higher nitrogenase activity compared to the reconstituted $v n f$ system with nifEN. However, the nifB2vnfHDGK operon of P. zanthoxyli JH29 can not complement the $\triangle$ nifBHDKEN mutant of $P$. polymyxa WLY78, suggesting that the $\operatorname{nfEN}$ or nifEN was required for the biosynthesis of VFe-co. The diazotrophic growth tests showed that all the reconstituted nifB/vnf-complemented strains excluding $\triangle$ nifBHDKEN/nifB2vnfHDGK strain grew as well as the $P$. zanthoxyli JH29 (Additional file 1: Figure S2). The results indicated that the reconstituted $v n f$ system composed of 10 genes (nifBvnfHDGK of P. zanthoxyli $\mathrm{JH} 29$ and nifENXhesAnifV of $P$. polymyxa WLY78 or nifBvnfHDGKEN of P. zanthoxyli JH29 and nifXhesAnifV of $P$. polymyxa WLY78) can support synthesis of $\mathrm{V}$-nitrogenase to fix nitrogen.

\section{Discussion}

Most of the diazotrophs carried a single copy of nifB. However, our results demonstrated that 2-4 nifB genes were distributed in Paenibacillus strains having additional nif genes or anf genes or $v n f$ genes. The occurrence of multiple nifB copies appears to be specific to diazotrophic Paenibacillus. In addition, the presence of nifB1 immediately upstream of the structural genes nifHDK and presence of nifB2 close to the structural genes anf$H D G K$ or $v n f H D G K$ also seem to characterize the genus. Our analyses have revealed that all nifB genes in Paenibacillus fall into 4 classes and their encoded products have a N-terminal SAM-radical domain linked to a $\mathrm{C}$-terminal NifX-like domain. However, the NifB3 protein of $P$. zanthoxyli JH29 or P. durus DSM 1735 is a stand-alone SAMradical protein which is adjacent to a NifX-like protein.
To confirm the accuracy of the nifB3 at DNA sequence level, a DNA fragment including both of the coding regions of a SAM-radical protein and a NifX-like protein was PCR amplified from P. zanthoxyli JH29 (Additional file 1: Figure S3). Sequence analysis have shown that the NifB3 protein of $P$. zanthoxyli JH29 is really a stand-alone SAM-radical protein that linked to a NifX-like protein. We deduce that the nifB3 gene of $P$. zanthoxyli $\mathrm{JH} 29$ or P. durus DSM 1735 is divided to two genes: one encoding a SAM-radical protein and the other encoding a NifXlike protein during evolution. The NifB proteins with only a SAM-radical domain are distributed in some bacteria and in most archaea [21]. However, a stand-alone SAM-radical domain in the NifB3 proteins of $P$. zanthoxyli JH29 and $P$. durus DSM 1735 lacks the C-terminal Cx2CRxDAxG motif that binds an Fe-S cluster necessary for NifB-co synthesis [29]. The NifB proteins with three domain architectures comprising a NifN-like domain, SAM-radical domain and a NifX domain are widely distributed in Clostridium genus [21]. However, the NifB proteins with three domain architectures are not found in Paenibacillus, although both Paenibacillus and Clostridium are genera of the Firmicutes phylum.

The canonical NifB protein contains a SAM-radical domain and a NifX-like domain. We have found that some $\mathrm{N}_{2}$-fixing Paenibacillus strains possess NifX-like protein that shows higher sequence similarity value with the C-terminal domain of NifB compared with that of NifX protein family. These proteins with only a NifXlike domain are also found in other diazotrophs, but they were eliminated from their studies [21]. Here, the transcription and function of the nifX-like genes from $P$. sabinae T27, P. forsythia T98 and P. zanthoxyli JH29 are investigated. Generally, the nifX-like gene in Paenibacillus strains is linked together with nifH or other gene. In $P$. sabinae T27, the nif $X$-like gene is located within the nifH nif $X$-like nif $N$ nifE cluster and is significantly transcribed under $\mathrm{N}_{2}$-fixing condition compared to non- $\mathrm{N}_{2}$-fixing condition (Additional file 1: Figure S4a). One possible reason is that the nif $X$-like and nifH were cotranscribed from a common promoter, consistent with previous studies that transcript of the nifH and nif $X$-like (previously called as nifB) increased under nitrogen fixation condition $[26,30]$. However, the transcription of nif $X$-like gene linked together with gldA gene in $P$. forsythia T98 or $P$. zanthoxyli $\mathrm{JH} 29$ was not upregulated under $\mathrm{N}_{2}$-fixing condition than non- $\mathrm{N}_{2}$-fixing condition (Additional file 1: Figure S4b, c). Complementation experiments demonstrate that NifX-like proteins of $P$. sabinae T27, $P$. forsythia T98 and $P$. zanthoxyli $\mathrm{JH} 29$ could not resume the nitrogenase activity of $P$. polymyxa $\triangle$ nifB mutant (Additional file 1: Figure S4d), indicating that these NifXlike proteins can not substitute NifB. It was reported that 
NifX-like domain of NifB is not required for nitrogen fixation but may perform complementary functions that are beneficial for FeMo-co biosynthesis [21].

Complementation studies revealed that either NifB1 or NifB2 protein can support any type of nitrogenase activity. However, expression analysis showed that nifB1 exhibited the greatest increase in expression under Modependent $\mathrm{N}_{2}$-fixing condition compared to alternative $\mathrm{N}_{2}$-fixing condition and nifB2 is even more induced under alternative $\mathrm{N}_{2}$-fixing condition compared to Modependent $\mathrm{N}_{2}$-fixing condition. This implies that the NifB1 or NifB2 are specifically expressed under different metal conditions to support synthesis of Mo- and alternative nitrogenases in original host cell, respectively. Some reports found that 2 nifB genes in diazotroph genomes $[18,20]$, but no further work has demonstrated their transcription levels under different metal conditions. It is reported that $P$. sabinae T27, $P$. zanthoxyli $\mathrm{JH} 29$ and P. forsythia T98 exhibited high nitrogenase activities compared to P. polymyxa WLY78 [31]. Previous studies showed that 3 nifH genes of $P$. sabinae T27 are functional by complementing $K$. oxytoca $\Delta$ nifH mutant [32]. Our present work demonstrated that nifB2 restored the nitrogenase activity of P. polymyxa WLY78 $\triangle$ nifB mutant. Thus, the higher nitrogenase activity exhibited by these species may be due to their additional nif genes.

The nifB3 and nifB4 were not exhibited higher transcriptional activity under $\mathrm{N}_{2}$-fixing condition than under non- $\mathrm{N}_{2}$-fixing condition, nor functionally complementing the most common and active nifB1 copy, and in some cases, displaying sequence divergence in regions of the protein already described as critical for NifB activity. Deletion analysis in the original Paenibacillus strain further revealed that nifB3 and nifB4 were not essential to nitrogen fixation. Thus, the nifB3 and nifB4 genes may be not functional or their genes products were inactive in synthesis of nitrogenase. They could be pseudogenes. Since the nifB3 and nifB4-encoded proteins exhibit sequence conservation with that of NifB1 and NifB2, transcription inactivity of nifB3 and nifB4 seems to be caused by mutations in their regulatory sequence, leading to prevent their expression.

Moreover, we extended the studies to reconstruct gene requirements for the alternative nitrogenase. Our current study has demonstrated that the reconstituted anf system composed of 8 genes (nifBanfHDGK and nifXhesAnifV) can support synthesis of Fe-nitrogenase to fix nitrogen in $P$. polymyxa. This is consistent with previous report that the nifEN is not required for the reconstruction Fe-nitrogenase in Escherichia coli [33]. In contrast, synthesis of V-nitrogenase is dependent on either nifEN or $v n f E N$. In A. vinelandii, NifEN can substitute for VnfEN in vnfEN mutants for the biosynthesis of VFe-co, but the VnfEN not NifEN is the preferred scaffold for FeV-co maturation $[34,35]$. Our result also confirms that VnfEN is more effective in FeV-co biosynthesis than NifEN.

Many efforts have been directed at engineering diazotrophic eukaryotes, one of the main hurdles is achieving NifB activity. Recent studies have found that the expressed NifB from the methanogen Methanocaldococcus infernus in the yeast cell was in a soluble form, while the expressed NifB from $A$. vinelandii in the yeast cells formed aggregates [36, 37]. In addition, the minimal number of genes required for nitrogen fixation is also the crucial step toward this goal. The Paenibacillus strains has some interesting features for engineering of eukaryotic $\mathrm{N}_{2}$ fixation, such as minimal nif gene cluster and additional nif and anf or $v n f$ genes. Our study may provide guidance for screening nif genes to sort the best candidates to generate efficient nitrogenase. Given widespread findings of terrestrial Mo limitation [38], the minimal Fe- nitrogenase and V- nitrogenase systems described here have practical potentials in engineering nitrogen fixing plants.

\section{Materials and methods \\ Phylogenetic analysis}

The 138 putative nifB gene sequences of the $116 \mathrm{~N}_{2}$-fixing Paenibacillus strains and 11 putative nifB gene sequences of 10 other diazotrophs (Frankia sp. EAN1pec, Nostoc sp. PCC7120, Bradyrhizobium japonicum USDA 6, Kyrpidia spormannii CVV65, Clostridium kluyveri DSM 555, Dehalobacter sp. CF, A. vinelandii DJ, K. oxytoca KONIH1, Methanococcus maripaludis S2 and Methanosarcina acetivorans $\mathrm{C} 2 \mathrm{~A}$ ) from the NCBI RefSeq database (last accessed July 2019) are shown in Table S1. Multiple alignment of amino acid sequences was performed by ClustalW (version 2.1) [39]. A maximum-likelihood phylogenetic tree of Paenibacillus species was constructed using PhyML (version 3.0) software [40].

\section{Plasmids, strains and growth conditions}

Strains and plasmids used in this work are listed in (Additional file 1: Table S2). Paenibacillus strains were routinely grown in $\mathrm{LD}$ medium $(2.5 \mathrm{~g} / \mathrm{L} \mathrm{NaCl}, 5 \mathrm{~g} / \mathrm{L}$ yeast and $10 \mathrm{~g} / \mathrm{L}$ tryptone) at $30^{\circ} \mathrm{C}$ with shaking under aerobic condition. For nitrogen fixation, Paenibacillus strains were grown in nitrogen-limited medium ( $0.3 \mathrm{~g} / \mathrm{L}$ glutamate) under anaerobic condition. Nitrogen-limited medium used in this study contains $10.4 \mathrm{~g} / \mathrm{L}$ of $\mathrm{Na}_{2} \mathrm{HPO} 4,3.4 \mathrm{~g} / \mathrm{L}$ of $\mathrm{KH}_{2} \mathrm{PO}_{4}, 26 \mathrm{mg} / \mathrm{L}$ of $\mathrm{CaCl}_{2} \cdot 2 \mathrm{H}_{2} \mathrm{O}, 30 \mathrm{mg} / \mathrm{L}$ of $\mathrm{MgSO}_{4}$, $0.3 \mathrm{mg} / \mathrm{L}$ of $\mathrm{MnSO}_{4}, 36 \mathrm{mg} / \mathrm{L}$ of Ferric citrate, $7.6 \mathrm{mg} / \mathrm{L}$ $\mathrm{Na}_{2} \mathrm{MoO}_{4} \cdot 2 \mathrm{H}_{2} \mathrm{O}, 10 \mu \mathrm{g} / \mathrm{L}$ of p-aminobenzoic acid, $5 \mu \mathrm{g} / \mathrm{L}$ of biotin, and $4 \mathrm{~g} / \mathrm{L}$ glucose, with $0.3 \mathrm{~g} / \mathrm{L}$ glutamate as the nitrogen source. Escherichia coli JM109 was used as routine cloning host. Thermo-sensitive vector pRN5101 [27, 
28] was used for gene disruption and complementation experiment in P. polymyxa WLY78 and P. sabinae T27. When appropriate, antibiotics were added in the following concentrations: $100 \mu \mathrm{g} / \mathrm{mL}$ ampicillin and $5 \mu \mathrm{g} / \mathrm{mL}$ erythromycin for maintenance of plasmids.

For diazotrophic growth, Paenibacillus strains and complementary strains were initially grown overnight in LD medium at $30^{\circ} \mathrm{C}$. Cells were collected, washed, and resuspended in nitrogen-free medium (nitrogen-limited medium without glutamate) under $\mathrm{N}_{2}$ atmosphere, with initial $\mathrm{OD}_{600}$ of 0.3 . After $48 \mathrm{~h}, \mathrm{OD}_{600}$ was detected.

\section{Acetylene reduction assays for nitrogenase activity}

Nitrogenase activity was measured by acetylene reduction assays as described previously [25]. For Mo-nitrogenase activity, P. polymyxa WLY78 and their derivatives were individually grown overnight in $50 \mathrm{~mL}$ of liquid $\mathrm{LD}$ media for $16 \mathrm{~h}$ at $30^{\circ} \mathrm{C}$ with shaking at $200 \mathrm{rpm}$. The culture was collected by centrifugation, and the pellet was washed three times with sterilized water and then resuspended in a $26 \mathrm{~mL}$ sealed tube containing $4 \mathrm{~mL}$ of nitrogen-limited medium to a final $\mathrm{OD}_{600}$ of 0.3 to 0.5 . The headspace in the tube was then evacuated and replaced with argon gas. After $\mathrm{C}_{2} \mathrm{H}_{2}$ (10\% of the headspace volume) was injected into the test tubes, the cultures were incubated at $30^{\circ} \mathrm{C}$ for $2-4 \mathrm{~h}$ and with shaking at $200 \mathrm{rpm}$. Then, $100 \mu \mathrm{L}$ of gas was withdrawn through the rubber stopper with a gas tight syringe and manually injected into the gas chromatograph HP6890 to quantify ethylene production. The nitrogenase activity was expressed in nmol $\mathrm{C}_{2} \mathrm{H}_{4} / \mathrm{mg}$ protein/hr. To assess Fe-nitrogenase activity, Mo-starved Paenibacillus cells were grown in nitrogen-limited medium that was depleted of molybdenum by Schneider et al. [41]. For V-nitrogenase activity, $30 \mu \mathrm{M} \mathrm{Na} \mathrm{VO}_{4}$ was added to the nitrogen-limited medium to take place of $\mathrm{Na}_{2} \mathrm{MoO}_{4}$. All treatments were in three replicates and all the experiments were repeated three or more than three times.

\section{Transcription analysis}

Transcription analyses of nifB genes were investigated by real-time quantitative PCR (RT-qPCR). Paenibacillus sabinae T27 was grown in nitrogen-limited medium containing $\mathrm{Mo}\left(\mathrm{Na}_{2} \mathrm{MoO} 4\right)$, while P. zanthoxyli $\mathrm{JH} 29$ and P. forsythia T98 were grown in Mo-free nitrogen-limited media containing Fe and V, respectively. For negative controls, these bacteria were individually grown in LD medium which has excess nitrogen medium to inhibit nitrogen fixation. These Paenibacillus strains were grown at $30^{\circ} \mathrm{C}$ with shaking under anaerobic condition. The bacterial cells were harvested after cultivation for $4 \mathrm{~h}$ cultivation. Total RNA was extracted with Trizol (Takara Bio, Tokyo, Japan) according to the manufacturer's instructions. The integrity and size distribution of the RNA was verified by agarose gel electrophoresis, and the concentration was determined spectrophotometrically. Remove of genome DNA and synthesis of cDNA were performed using RT Prime Mix according to the manufacturer's specifications (Takara Bio, Tokyo, Japan). Primers for nif genes and 16S rDNA used for RT-qPCR are listed in Additional file 1: Table S3. RT-qPCR was performed on Applied Biosystems 7500 Real-Time System and detected by the SYBR Green detection system with the following program: $95^{\circ} \mathrm{C}$ for $15 \mathrm{~min}, 1 \mathrm{cycle} ; 95^{\circ} \mathrm{C}$ for $10 \mathrm{~s}$ and $65^{\circ} \mathrm{C}$ for $30 \mathrm{~s}, 40$ cycles. The relative expression level was calculated using the $2^{-\Delta \Delta \mathrm{CT}}$ method [42]. Each experiment was performed in triplicate.

\section{Construction of $\triangle$ nifB, $\triangle$ nifBHDK and $\triangle$ nifBHDKEN mutants of $P$. polymyxa}

The nifB, nifBHDK and nifBHDKEN deletion mutants of P. polymyxa WLY78 were constructed by a homologous recombination method. The upstream (ca. $1 \mathrm{~kb}$ ) and downstream (ca. $1.0 \mathrm{~kb}$ ) fragments flanking the coding region of $n i f B$ or $n i f B H D K$ or nifBHDKEN were amplified by PCR from the genomic DNA of P. polymyxa WLY78 using Super-Fidelity DNA Polymerase (Vazyme Biotech Co., Ltd., Nanjing, China), respectively. The two fragments flanking coding region of nifB or nifBHDK or nifB$H D K E N$ were then fused with $B a m H$ I digested pRN5101 vector using Gibson assembly master mix (New England Biolabs, Ipswich, USA), generating the recombinant plasmids pRDnifB, pRDnifBHDK and pRDnifBHDKEN, respectively. Then, each of these recombinant plasmids was transformed into P. polymyxa WLY78 as described by Wang et al., [43]. Subsequently, marker-free deletion mutants (the double-crossover transformants) $\triangle$ nifB, $\triangle n i f B H D K$ and $\triangle n i f B H D K E N$ were selected from the initial $\mathrm{Em}^{\mathrm{r}}$ transformants after several rounds of nonselective growth at $39{ }^{\circ} \mathrm{C}$ and then confirmed by PCR amplification and sequencing analysis. The primers used for the PCR amplifications were listed in Additional file 1: Table S3.

\section{Construction of plasmids for complementation of the $P$. polymyxa $\triangle$ nifB mutant}

Here, 9 nifB genes from $P$. sabinae T27, P. forsythia T98 and $P$. zanthoxyli $\mathrm{JH} 29$ were used to complement the $P$. polymyxa $\triangle$ nifB mutant. These nifB genes include nifB1, nifB3 and nifB4 of $P$. sabinae T27, nifB1, nifB2 and nifB3 of $P$. forsythia T98 and nifB1, nifB2 and nifB3 of $P$. zanthoxyli JH29. The coding region of each nifB gene from $P$. sabinae T27, P. forsythia T98 and P. zanthoxyli JH29 and a 310 bp promoter region of nifB in the nifBHDKENXhesAnifV operon of P. polymyxa WLY78 were PCR amplified. Then, The PCR products of the nifB coding region 
and the promoter region were fused together with vector pRN5101 using Gibson assembly master mix, yielding the recombinant plasmid. The recombinant plasmid was transformed to P. polymyxa WLY78 nifB mutant for complementation. The primers used in fusion were listed in Additional file 1: Table S3.

\section{Construction of $\triangle$ nifB1, $\Delta$ nifB3 and $\triangle$ nifB4 mutants of $P$. sabinae $\mathrm{T} 27$ and complementation strain}

Three nifB deletion mutants in $P$. sabinae T27 including $\triangle n i f B 1, \triangle n i f B 3$ and $\triangle$ nifB4 were constructed via homologous recombination using the suicide plasmid pRN5101 as described above. The upstream and downstream fragments flanking the coding region of nifB1, nifB3 and nifB4 were PCR amplified from the genomic DNA of $P$. sabinae T27, respectively. The primers used for deletion mutagenesis are listed in Additional file 1: Table S3. The upstream and downstream fragments of three nifB genes were then fused with $B a m H$ I -digested vector pRN5101 in Gibson assembly master mix, generating the three recombinant plasmids pRDnifB1, pRDnifB3 and $\mathrm{pRDnifB} 4$. Then, each of these recombinant plasmids was electroporated into P. sabinae T27, and the deletion mutants were screened and confirmed by PCR and sequencing.

For complementation of $\triangle$ nifB1, a DNA fragment carrying the nifB1 ORF (1377 bp) and its own promoter (549 bp) was PCR amplified and then ligated to pRN5101 and then transformed to $P$. sabinae T27 $\triangle$ nifB1, generating the nifB1 complemented strain nifB1/nifB1. The primers used here are listed in Additional file 1: Table S3.

\section{Construction of the recombinant plasmids for complementation of the P. polymyxa $\triangle$ nifBHDK or $\triangle$ nifBHDKEN mutant}

For construction recombinant plasmids of alternative nitrogenases in $P$. polymyxa, the coding regions of the nifB1, nifB2, the anfHDGK and nifE2N2anfHDGK operon were amplified from the genome of $P$. forsythia T98, respectively. Also, a $310 \mathrm{bp}$ promoter region of nifB in the nifBHDKENXhesAnifV operon of P. polymyxa WLY78 was PCR amplified. Then, the PCR amplified promoter, nifB1 or nifB2 and the anfHDGK or nifE2N2anfHDGK operon were in order linked to vector pRN5101 using Gibson assembly master mix, yielding the recombinant plasmid carrying the reconstituted nifB1anfHDGK operon or nifB2anfHDGK operon or nif$B 2 E 2 N 2 a n f H D G K$ operon. The expression of nifB1vnfH$D G K$ or nifB2vnfHDGK or nifE2N2anfHDGK was under control of the $P$. polymyxa nifB promoter. Finally, these plasmids were individually transformed into $\triangle$ nifBHDK or $\triangle$ nifBHDKEN mutant of P. polymyxa WLY78.
Similarly, the nifB1, nifB2, vnfHDGK and vnfHDG$K E N$ operon were amplified from the genome of $P$. zanthoxyli $\mathrm{JH} 29$, respectively. A $310 \mathrm{bp}$ promoter region of nifB in the nifBHDKENXhesAnifV operon of P. polymyxa WLY78 was PCR amplified. Then, the three fragments including the promoter, nifB1 or nifB2 and $v n f H D G K$ or $v n f H D G K E N$ operon were in order fused together with vector pRN5101 using Gibson assembly master mix, yielding the recombinant plasmid carrying the reconstituted operon nifB1vnfHDGK or nifB2vnfHDGK or nifB2vnfHDGKEN. The expression of nifB1vnfHDGK or nifB2vnfHDGK or nifB2vnfHDGKEN was under control of the $P$. polymyxa nifB promoter. Finally, these plasmids were individually transformed into $\triangle$ nifBHDK mutant or $\triangle$ nifBHDKEN of P. polymyxa WLY78.

\section{Supplementary Information}

The online version contains supplementary material available at https://doi. org/10.1186/s12934-021-01629-9.

Additional file 1: Table S1. The nifB gene in diazotrophic Paenibacillus strains and other representative diazotrophs. Figure S1. Sequence alignment of 10 NifB proteins and 3 NifX-like proteins from 4 representatives of $\mathrm{N}_{2}$-fixing Paenibacillus strains (P. polymyxa WLY78, P. sabinae T27, P. forsythia T98 and P. zanthoxyli JH29). Figure S2. Diazotrophic growth of the $\triangle$ nifBHDK and $\triangle$ nifBHDKEN mutants of P. polymyxa and the complementary strains carrying nifB1anfHDGK, nifB2anfBHDGK, nifB2E2N2anfBHDGK from P. forsythia T98, and nifB1vnfHDGK, nifB2vnfHDGK, nifB2vnfHDGKEN from P. zanthoxyli JH29, respectively. Figure S3. Nucleotide sequence of DNA fragment containing nifB3 and an additional nifX-like in P. zanthoxyli JH29 and $P$. durus DSM 1735. Figure S4. Transcription analysis of the nif $X$-like genes and nitrogenase activities of the P. polymyxa $\triangle$ nifB complementary strains carrying nifX-like genes under nitrogen fixation conditions. Table S2. Bacterial strains and plasmids used in this study. Table S3. Primers used for RT-qPCR, construction of nifB, nifBHDK, nifNBHDKEN, nifB1, nifB3, nifB4 mutants and complementation strains.

Acknowledgements

We thank Dr. Sishuo Wang for his guidance in phylogenetic analysis and helpful discussion.

\section{Authors' contributions}

QL performed all experiments, and drafted the manuscript. HWZ participated in strain construction. LQZ assisted in the writing. SFC conceived the study, guided its coordination and wrote the manuscript. All authors read and approved the final manuscript.

\section{Funding}

This work was supported by the National Natural Science Foundation of China (No. 32000048) and the National Key Research and Development Program of China (No. 2019YFA0904700).

\section{Availability of data and materials}

All data generated or analysed during this study are included in this published article and are available from the corresponding author on reasonable request.

\section{Declarations}

Ethics approval and consent to participate Not applicable. 


\section{Consent for publication \\ Not applicable.}

\section{Competing interests}

The authors declare no competing interests.

\section{Author details}

${ }^{1}$ State Key Laboratory for Agrobiotechnology, College of Biological Sciences, China Agricultural University, Beijing 100193, People's Republic of China. ${ }^{2}$ Key Laboratory of Pest Monitoring and Green Management, Ministry of Agriculture and Rural Affairs, and College of Plant Protection, China Agricultural University, Beijing 100193, People's Republic of China.

\section{Received: 19 April 2021 Accepted: 10 July 2021}

Published online: 19 July 2021

\section{References}

1. Dos Santos PC, Fang Z, Mason SW, Setubal JC, Dixon R. Distribution of nitrogen fixation and nitrogenase-like sequences amongst microbial genomes. BMC Genomics. 2012;13:162.

2. Ribbe MW, Hu YL, Hodgson KO, Hedman B. Biosynthesis of nitrogenase metalloclusters. Chem Rev. 2014;114:4063-80.

3. Eady RR. Structure-function relationships of alternative nitrogenases. Chem Rev. 1996;96:3013-30.

4. Rubio LM, Ludden PW. Biosynthesis of the iron-molybdenum cofactor of nitrogenase. Annu Rev Microbiol. 2008;62:93-111.

5. Mus F, Alleman AB, Pence N, Seefeldt LC, Peters JW. Exploring the alternatives of biological nitrogen fixation. Metallomics. 2018;10:523-38.

6. Hu YL, Ribbe MW. Biosynthesis of the metalloclusters of molybdenum nitrogenase. Microbiol Mol Biol Rev. 2011;75:664-77.

7. Hoffman BM, Lukoyanov D, Yang ZY, Dean DR, Seefeldt LC. Mechanism of nitrogen fixation by nitrogenase: the next stage. Chem Rev. 2014;114:4041-62.

8. Buren S, Jimenez-Vicente E, Echavarri-Erasun C, Rubio LM. Biosynthesis of nitrogenase cofactors. Chem Rev. 2020;120:4921-68.

9. McRose DL, Zhang XN, Kraepiel AML, Morel FMM. Diversity and activity of alternative nitrogenases in sequenced genomes and coastal environments. Front Microbiol. 2017;8:267.

10. Harwood CS. Iron-only and vanadium nitrogenases: fail-safe enzymes or something more? Annu Rev Microbiol. 2020;74:247-66.

11. Sippel D, Einsle $O$. The structure of vanadium nitrogenase reveals an unusual bridging ligand. Nat Chem Biol. 2017:13:956-61.

12. Curatti L, Ludden PW, Rubio LM. NifB-dependent in vitro synthesis of the iron-molybdenum cofactor of nitrogenase. Proc Natl Acad Sci U S A. 2006;103:5297-301

13. Wiig JA, Hu YL, Lee CC, Ribbe MW. Radical SAM-dependent carbon insertion into the nitrogenase M-cluster. Science. 2012;337:1672-5.

14. Fajardo AS, Legrand P, Paya-Tormo LA, Martin L, Pellicer Marti Nez MT, Echavarri-Erasun C, Vernede X, Rubio LM, Nicolet Y. Structural insights into the mechanism of the Radical SAM carbide synthase NifB, a key nitrogenase cofactor maturating enzyme. J Am Chem Soc. 2020;142:11006-12.

15. Hernandez JA, Igarashi RY, Soboh B, Curatti L, Dean DR, Ludden PW, Rubio LM. NifX and NifEN exchange NifB cofactor and the VK-cluster, a newly isolated intermediate of the iron-molybdenum cofactor biosynthetic pathway. Mol Microbiol. 2007:63:177-92.

16. Drummond M, Walmsley J, Kennedy C. Expression from the nifB promoter of Azotobacter vinelandii can be activated by NifA, VnfA, or AnfA transcriptional activators. J Bacteriol. 1996;178:788-92.

17. Oda Y, Samanta SK, Rey FE, Wu LY, Liu XD, Yan TF, Zhou JZ, Harwood CS. Functional genomic analysis of three nitrogenase isozymes in the photosynthetic bacterium Rhodopseudomonas palustris. J Bacteriol. 2005:187:7784-94

18. Demtroder L, Narberhaus F, Masepohl B. Coordinated regulation of nitrogen fixation and molybdate transport by molybdenum. Mol Microbiol. 2019;111:17-30

19. Schuddekopf K, Hennecke S, Liese U, Kutsche M, Klipp W. Characterization of anf genes specific for the alternative nitrogenase and identification of nif genes required for both nitrogenases in Rhodobacter capsulatus. Mol Microbiol. 1993:8:673-84.
20 Vernon SA, Pratte BS, Thiel T. Role of the nifB1 and nifB2 promoters in eell-type-specific expression of two Mo nitrogenases in the Cyanobacterium Anabaena variabilis ATCC 29413. J Bacteriol. 2017. https://doi.org/10. 1128/JB.00674-16.

21. Arragain S, Jimenez-Vicente E, Scandurra AA, Buren S, Rubio LM, Echavarri-Erasun C. Diversity and functional analysis of the FeMo-cofactor maturase NifB. Front Plant Sci. 2017:8:1947.

22. Boyd ES, Anbar AD, Miller S, Hamilton TL, Lavin M, Peters JW. A late methanogen origin for molybdenum-dependent nitrogenase. Geobiology. 2011;9:221-32

23. Grady EN, MacDonald J, Liu L, Richman A, Yuan ZC. Current knowledge and perspectives of Paenibacillus: a review. Microb Cell Fact. 2016;15:203.

24. Xie JB, Du ZL, Bai LQ, Tian CF, Zhang YZ, Xie JY, Wang TS, Liu XM, Chen X Cheng Q, Chen SF, Li JL. Comparative genomic analysis of $\mathrm{N}_{2}$-fixing and non- $\mathrm{N}_{2}$-fixing Paenibacillus spp.: organization, evolution and expression of the nitrogen fixation genes. Plos Genet. 2014;10:e1004231.

25. Wang LY, Zhang LH, Liu ZZ, Zhao DH, Liu XM, Zhang B, Xie JB, Hong YY, Li PF, Chen SF, Dixon R, Li JL. A minimal nitrogen fixation gene cluster from Paenibacillus sp. WLY78 enables expression of active nitrogenase in Escherichia coli. Plos Genet. 2013:9:e1003865.

26. Li XX, Deng ZP, Liu ZZ, Yan YL, Wang TS, Xie JB, Lin M, Cheng Q, Chen SF. The genome of Paenibacillus sabinae $\mathrm{T} 27$ provides insight into evolution, organization and functional elucidation of nif and nif-like genes. BMC Genomics. 2014;15:723.

27. Villafane R, Bechhofer DH, Narayanan CS, Dubnau D. Replication control genes of plasmid pE194. J Bacteriol. 1987;169:4822-9.

28. Zhang W, Ding Y, Yao L, Liu K, Du B. Construction of gene knock-out system for Paenibacillus polymyxa SC2. Acta Microbiol Sin. 2013;53:1258-66.

29. Kang W, Rettberg LA, Stiebritz MT, Jasniewski AJ, Tanifuji K, Lee CC, Ribbe MW, Hu Y. X-ray crystallographic analysis of NifB with a full complement of clusters: structural insights into the radical SAM-dependent carbide insertion during nitrogenase cofactor assembly. Angew Chem Int Edit. 2021;60:2364-70.

30. Li Q, He X, Liu P, Zhang H, Wang M, Chen S. Synthesis of nitrogenase by Paenibacillus sabinae T27 in presence of high levels of ammonia during anaerobic fermentation. Appl Microbiol Biot. 2021;105:2889-99.

31 Ma YC, Xia ZQ, Liu XM, Chen SF. Paenibacillus sabinae sp. nov., a nitrogenfixing species isolated from the rhizosphere soils of shrubs. Int J Syst Evol Microbiol. 2007;57:6-11

32. Hong YY, Ma YC, Wu LX, Maki M, Oin WS, Chen SF. Characterization and analysis of nifH genes from Paenibacillus sabinae T27. Microbiol Res. 2012;167:596-601.

33. Yang JG, Xie XQ, Wang X, Dixon R, Wang YP. Reconstruction and minimal gene requirements for the alternative iron-only nitrogenase in Escherichia coli. Proc Natl Acad Sci U S A. 2014;111:E3718-25.

34. Wolfinger ED, Bishop PE. Nucleotide sequence and mutational analysis of the $v$ VIENX region of Azotobacter vinelandii. J Bacteriol. 1991;173:7565-72.

35. Hamilton TL, Ludwig M, Dixon R, Boyd ES, Dos Santos PC, Setubal JC, Bryant DA, Dean DR, Peters JW. Transcriptional profiling of nitrogen fixation in Azotobacter vinelandii. J Bacteriol. 2011;193:4477-86.

36. Buren S, Jiang X, Lopez-Torrejon G, Echavarri-Erasun C, Rubio LM. Purification and in vitro activity of mitochondria targeted nitrogenase cofactor maturase NifB. Front Plant Sci. 2017:8:1567.

37. Buren S, Pratt K, Jiang X, Guo Y, Jimenez-Vicente E, Echavarri-Erasun C, Dean DR, Saaem I, Gordon DB, Voigt CA, Rubio LM. Biosynthesis of the nitrogenase active-site cofactor precursor NifB-co in Saccharomyces cerevisiae. Proc Natl Acad Sci U S A. 2019:116:25078-86.

38. Darnajoux R, Magain N, Renaudin M, Lutzoni F, Bellenger JP, Zhang $X N$. Molybdenum threshold for ecosystem scale alternative vanadium nitrogenase activity in boreal forests. Proc Natl Acad Sci U S A. 2019;116:24682-8.

39 Thompson JD, Gibson TJ, Higgins DG. Multiple sequence alignment using ClustalW and ClustalX. Current Protoc Bioinformatics. 2002. https://doi. org/10.1002/0471250953.bi0203s00.

40 Guindon S, Dufayard JF, Lefort V, Anisimova M, Hordijk W, Gascuel O. New algorithms and methods to estimate maximum-likelihood phylogenies: assessing the performance of PhyML 3.0. Syst Biol. 2010;59:307-21.

41. Schneider K, Muller A, Johannes KU, Diemann E, Kottmann J. Selective removal of molybdenum traces from growth media of $\mathrm{N}_{2}$-fixing bacteria. Anal Biochem. 1991;193:292-8. 
42. Livak KJ, Schmittgen TD. Analysis of relative gene expression data using real-time quantitative PCR and the $2^{-\triangle \Delta C T}$ method. Methods. 2001;25:402-8.

43. Wang TS, Zhao XY, Shi HW, Sun L, Li YB, Li Q, Zhang HW, Chen SF, Li JL. Positive and negative regulation of transferred nif genes mediated by indigenous GInR in Gram-positive Paenibacillus polymyxa. Plos Genet. 2018:14:e1007629.

\section{Publisher's Note}

Springer Nature remains neutral with regard to jurisdictional claims in published maps and institutional affiliations.
Ready to submit your research? Choose BMC and benefit from:

- fast, convenient online submission

- thorough peer review by experienced researchers in your field

- rapid publication on acceptance

- support for research data, including large and complex data types

- gold Open Access which fosters wider collaboration and increased citations

- maximum visibility for your research: over 100M website views per year

At BMC, research is always in progress.

Learn more biomedcentral.com/submissions 